\section{Of stars}

\section{and planets}

David W. Hughes

100 Billion Suns: The Birth, Life and

Death of the Stars.

By Rudolf Kippenhahn.

Basic Books, New York/Weidenfeld and

Nicolson: 1983. Pp.264 \$25, f15.

The New Solar System (2nd Edition).

Edited by J. Kelly Beatty, Brian

O'Leary and Andrew Chaikin.

Cambridge University Press: 1983.

Pp.240. f12.50, \$24.95.

EJNAR Hertzsprung and Henry Norris Russell provided one of the most fruitful avenues of astrophysical display when they independently plotted the colours (or spectra) of stars as abscissae against their absolute magnitudes (or luminosities) as ordinate. This $\mathrm{H}-\mathrm{R}$ diagram has become the backbone of all discussions of stellar evolution, and its main sequence and giant branch are the temporary dwelling places of the large majority of stars. The tortuous path of a star around this diagram illustrates graphically the change in size, surface temperature and energy generation process that occur to a star of a specific mass as it evolves.

Many of the most fascinating problems of astrophysics are enshrined in this evolution. Why do some clouds collapse to form stars, when and how do stars change nuclear fuels from hydrogen to helium, what is the role of mass loss in evolution, what is the dividing line between white dwarfs and neutron stars, pulsars and $\mathrm{X}$-ray sources, why do some stars become Cepheids?

Kippenhahn has spent the large majority of his research career investigating stellar evolution using sophisticated computer models to represent the physical characteristics and nuclear processes in the interior of stars. To him stars are not merely phenomena inviting our awestruck admiration. They are objects of our universe and as such are subject to the law of physics. Chapter by chapter he unravels their intricacies. At the end the reader is left with a vivid understanding of how stars work.

The reader is continuously aware that the author has had first hand experience of the subject. It is also obvious that many of the astronomers mentioned in the book are the personal friends and acqaintances of Rudolph Kippenhahn and his anecdotes and insights into the workings of this branch of astrophysics are fascinating. The illustrations and figures are excellent. The non-mathematical exposition is clear and precise and this book is an admirable introduction to the difficult subject of stellar evolution accurately aimed at the general reader. The book under review is a translation of the German edition which was published in 1980 (Piper \& Co., Munich).
Only fifteen months separate the first and second edition of The New Solar System but fifteen months is a long time in planetology. Voyager 2 has rushed past Saturn and two Soviet spacecraft have plummeted through the clouds to land on the surface of Venus. The 'average scientist' has been to two or three conferences in that time and written half a dozen or more research papers. There is plenty to update.

The first edition contained twenty chapters each of about ten pages. The authors were American, the list reading like a 'Who's Who' of solar system experts. Typical examples are Van Allen on magnetospheres and the interplanetary medium, Shoemaker on cratering, Masursky on Mars, Chapman on asteroids, Burns on rings, Pollack on Titan and Wood on meteorites. No change in chapter size has been allowed in the new edition. The only major difference between the two is the append-

\section{Making waves}

\section{F.A. Dahlen}

\section{Seismic Wave Propagation in} Stratified Media.

By B.L.N. Kennett.

Cambridge University Press: 1983.

Pp.342. £30. \$59.50.

IT HAS been said there are two good times to write a specialized book on a scientific topic. One is when a new field is just beginning to open up and a well-written treatise can pave the way for future research; the other is when a field is mature and fully developed, ripe for its elegance and unity to be revealed.

Kennett's account of the theory of seismic wave propagation in stratified media is a superb example of a book in the second category. Spurred by a number of diverse impetuses, the construction of synthetic seismograms to aid in structural and seismic source studies has progressed rapidly during the past two decades. Kennett has summarized this progress just at a time when the methods have become fairly well established and the attention of many seismologists is turning away from stratified media towards the investigation of lateral heterogeneity on a variety of scales.

When first asked if I would review this book, I considered my already crowded schedule and thought I should decline in favour of a more knowledgeable colleague. I'm glad I didn't because I learned a great deal by taking the time to read it slowly and carefully. Kennett begins by setting up the matrix framework appropriate to stratified media, following this with a brief discussion of seismic point sources. ing of a 14 page map section. Just imagine the U.S. Geological Survey map of Mercury miniaturized to fit into an area 17 $x 23 \mathrm{~cm}$ and you quickly realize that people without a handy magnifying glass will find these maps of very limited use. Most of the lettering is unreadable.

However, people will not buy this book for the maps but they should buy it, because it is well written, full of superb photographs, beautiful artists impressions and excellent figures. It is a first rate introduction to the solar system and a book against which popular astronomy books of the future will be measured. Too little has changed to make it imperative for owners of the first edition to buy the new one. But if you haven't got edition one, and have the slightest glimmer of an interest in the solar system, get this book.

David W Hughes is a lecturer in Astronomy and Physics at the University of Sheffield.

The main unifying theme is introduced next in two long (and occasionally difficult) chapters on the reflection and transmission properties of the stratification. The subsequent chapters describe both phase and slowness methods for constructing complete theoretical seismograms, as well as less-expensive systematic approximations to the full response useful in many applications. The emphasis throughout is on planar stratification, with occasional paragraphs or brief sections on the extension to the spherical case. All the important recent developments due to Chapman, Fuchs, Kennett himself, Müller, Richards, Woodhouse and others are clearly described here and compared in the context of the reflection matrix approach.

The usual high production standards of the Cambridge Monographs on Mechanics and Applied Mathematics have been maintained, although I did detect a fair number of typographical errors and omissions. Kennett is to be congratulated in particular for devising a consistent and reasonably self-explanatory notation, but a glossary would have been helpful - I frequently found myself thumbing back through to remember the difference between $\mathbf{R}_{L}^{\text {rs }}$ and $\mathbf{R}$, or the meaning of $\tilde{\mathbf{w}}$.

In summary, this is a model mathematical treatise, written with authority by an expert but accessible with diligence to all seismologists. It would be an excellent basis for a topical seminar on advanced seismology. Together with Aki and Richards's Quantitative Seismology (W.H. Freeman, 1980), the book certainly should be mandatory reading for theoretical seismologists for some time to come.

F.A. Dahlen is a Professor of Geophysics in the Department of Geological and Geophysical Sciences, Princeton University. 